\title{
On Existence and Asymptotic Behavior of Solutions of Hadamard-Volterra Integral Equations
}

\author{
Said Baghdad and Mouffak Benchohra*
}

\begin{abstract}
In this paper we provide sufficient condition guaranteeing existence and the asymptotic behavior of solutions of a class of Hadamard-Volterra integral equations in the Banach space of continuous and bounded functions on unbounded interval. The main tools used in our considerations are the concept of measure of noncompactness in conjunction with the Darbo and Mönch fixed point theorems.

Keywords: Hadamard-Volterra integral equations; Fixed-point theorems; Measure of noncompactness; Asymptotic behavior. AMS Subject Classification (2010): Primary: 34A08 ; Secondary: 34A08; $45 G 05$.

*Corresponding author
\end{abstract}

\section{Introduction}

The theory of integral operators and integral equations is an important part of nonlinear analysis. It is caused by the fact that this theory is frequently applicable in other branches of mathematics and mathematical physics, engineering, economics, biology as well in describing problems connected with real world see [7, 10, 11, 19, 23]. Fractional calculus is a generalization of the ordinary differentiation and integration to arbitrary non-integer order, it has developed up to the present day [7]. Differential and integral equations of fractional order are one of the most useful mathematical tools in both pure and applied analysis, and various theoretical results have been obtained, see $[1-5,17]$. The paper is devoted to the study of a class of integral equations of Hadamard-Volterra type. That class comprises a lot of particular cases of fractional integral equations which can be encountered in research papers and monographs concerning the theory of integral equations and their applications to real world problems see $[8,15,21,22,24]$. Consider the following integral equation

$$
u(x, y)=\mu(x, y)+\frac{1}{\Gamma\left(r_{1}\right) \Gamma\left(r_{2}\right)} \int_{1}^{x} \int_{1}^{y}\left(\ln \frac{x}{s}\right)^{r_{1}-1}\left(\ln \frac{y}{t}\right)^{r_{2}-1} \frac{f(t, s, u(t, s))}{s t} d t d s,
$$

where $(t, s) \in J=[1,+\infty) \times[1, b], r_{1}, r_{2}>0, \mu: J \rightarrow \mathbb{R}$ is a continuous and bounded function, $f: J \times \mathbb{R} \rightarrow \mathbb{R}$ is a continuous function, and $\Gamma(\cdot)$ is the Euler gamma function.

Using the technique associated with measures of noncompactness and fixed point theorems we show that Eq-(1.1) has solutions being continuous and bounded functions on the interval $[1,+\infty)$. Moreover, the choice of suitable measures of noncompactness allows us to assert that those solutions are asymptotically stable in certain sense which will be defined in the sequel.

\section{Preliminaries}

This section is devoted to collect some definitions and auxiliary results which will be needed in further considerations. At the beginning we present some basic facts concerning measures of noncompactness. Assume that $(X,\|\cdot\|)$ is an infinite dimensional Banach space with zero element $\theta$. Denote by $B(x, r)$ the closed ball centered

Received : 08-05-2018, Accepted : 11-02-2019 
at $x$ and radius $r$. We write $B_{r}$ to denote the ball $B(\theta, r)$. If $A$ is a subset of $X$ then the symbols $\bar{A}$, Conv $A$ stand for the closure and closed convex hull of $A$, respectively. Moreover, we denote by $\mathrm{M}_{X}$ the family of all nonempty and bounded subsets of $X$ and by $\mathrm{N}_{X}$ its subfamily consisting of all relatively compact sets. We accept the following definition of the concept of a measure of noncompactness.

Definition 2.1. $[13,15,16,18]$ A mapping $\psi: \mathrm{M}_{X} \rightarrow \mathbb{R}_{+}$is said to be a measure of noncompactness in $X$ if it satisfies the following conditions

1. The family ker $\psi=\left\{A \in \mathrm{M}_{X} / \psi(A)=0\right\}$ is nonempty and $\operatorname{ker} \psi \subset \mathrm{N}_{X}$.

2. $A \subset B \Rightarrow \psi(A) \leq \psi(B)$.

3. $\psi(A)=\psi(\bar{A})$.

4. $\psi(A)=\psi(\operatorname{Conv} A)$.

5. $\psi(\lambda A+(1-\lambda) B) \leq \lambda \psi(A)+(1-\lambda) \psi(B)$ for $\lambda \in[0,1]$.

6. If $\left(A_{n}\right)$ is a sequence of closed sets from $\mathrm{M}_{X}$ such that $A_{n+1} \subset A_{n}(n=0,1, \ldots)$ and if $\lim _{n \longrightarrow \infty} \psi\left(A_{n}\right)=0$, then the intersection set $A_{\infty}=\bigcap_{n=0}^{\infty} A_{n}$ is nonempty.

The family ker $\psi$ described in 1. is said to be the kernel of the measure of noncompactness $\psi$.

Remark 2.1. Observe that the intersection set $A_{\infty}$ from 6. is a member of the family ker $\psi$. In fact, since $\psi\left(A_{\infty}\right) \leq$ $\psi\left(A_{n}\right)$ for any $n$, we infer that $\psi\left(A_{\infty}\right)=0$.

In what follows we will work in the Banach space $X=B C(J)$ consisting of all real functions defined, continuous and bounded on $J$. This space is furnished with the standard norm

$$
\|u\|=\sup \{|u(x, y)| ;(x, y) \in J\} .
$$

In order to define a measure of noncompactness in the space $X$, let us fix a nonempty bounded subset $Y$ of the space $X$. For $u \in Y, T \geq 1, \epsilon_{1}, \epsilon_{2}>0,\left(x_{1}, y_{1}\right),\left(x_{2}, y_{2}\right) \in[1, T] \times[1, b]$ such that $\left|x_{2}-x_{1}\right| \leq \epsilon_{1}$ and $\left|y_{2}-y_{1}\right| \leq \epsilon_{2}$. We denote by $\omega^{T}\left(u, \epsilon_{1}, \epsilon_{2}\right)$ the modulus of continuity of the function $u$ on the interval $[1, T] \times[1, b]$ i.e

$$
\begin{aligned}
\omega^{T}\left(u, \epsilon_{1}, \epsilon_{2}\right) & =\sup \left\{\left|u\left(x_{2}, y_{2}\right)-u\left(x_{1}, y_{1}\right)\right| ;\left(x_{1}, y_{1}\right),\left(x_{2}, y_{2}\right) \in[1, T] \times[1, b]\right\} \\
\omega^{T}\left(Y, \epsilon_{1}, \epsilon_{2}\right) & =\sup \left\{\omega^{T}\left(u, \epsilon_{1}, \epsilon_{2}\right) ; u \in Y\right\} \\
\omega_{0}^{T}(Y) & =\lim _{\epsilon_{1}, \epsilon_{2} \rightarrow 0} \omega^{T}\left(Y, \epsilon_{1}, \epsilon_{2}\right) \\
\omega_{0}(Y) & =\lim _{T \rightarrow \infty} \omega_{0}^{T}(Y) .
\end{aligned}
$$

If $(t, s)$ is a fixed number from $J$, let us denote $Y(t, s)=\{u(t, s) ; u \in Y\}$ and

$$
\operatorname{diam} Y(t, s)=\sup \{|u(t, s)-v(t, s)| ; u, v \in Y\} .
$$

Finally, consider the function $\psi$ defined on the family $\mathrm{M}_{X}$ by the formula

$$
\psi(Y)=\omega_{0}(Y)+\lim _{t \longmapsto \infty} \sup \operatorname{diam} Y(t, s) .
$$

It can be shown that the function $\psi$ is a measure of noncompactness in the space $X$. The kernel ker $\psi$ consists of nonempty and bounded sets $Y$ such that functions from $Y$ are locally equicontinuous on $J$ and the thickness of the bundle formed by functions from $Y$ tends to zero at infinity. This property will permit us to characterize solutions of the integral equation considered in the next section.

Theorem 2.1. (Darbo)[14]

Let $\Omega$ be a nonempty, bounded, closed and convex subset of the Banach space $E$ and let $F: \Omega \longrightarrow \Omega$ be a continuous mapping. Assume that there exists a constant $k \in[0,1)$ such that $\psi(F A) \leq k \psi(A)$ for any nonempty subset $A$ of $\Omega$. Then $F$ has a fixed point in the set $\Omega$.

Remark 2.2. Let us denote by Fix $F$ the set of all fixed points of the operator $F$ which belong to $\Omega$. It can be shown that the set Fix $F$ belongs to the family $\operatorname{ker} \psi$. 
Theorem 2.2. (Mönch) $[18,20]$

Let $D$ be a bounded, closed and convex subset of the Banach space $E$ such that $0 \in D$, and let $F: D \longrightarrow D$ be a continuous mapping. If the implication

$$
V=\overline{\operatorname{Conv}} F(V) \quad \text { or } \quad V=F(V) \cup\{0\} \Rightarrow \psi(V)=0,
$$

holds for every subset $V$ of $D$. Then $F$ has a fixed point.

Now, let us assume that $\Omega$ is a nonempty subset of the space $X$ and $F$ is an operator on $\Omega$ with values in $X$. Consider the following equation

$$
u(x, y)=(F u)(x, y) ;(x, y) \in J .
$$

Definition 2.2. $[9,10,12]$ The solution $u=u(x, y)$ of Eq. (2.2) is said to be globally attractive if for each solution $v=v(x, y)$ of Eq. (2.2) we have that

$$
\lim _{x \longmapsto \infty}(u(x, y)-v(x, y))=0 .
$$

In the case when this limit is uniform, i.e., when for each $\epsilon>0$ there exists $T>0$ such that

$$
|u(x, y)-v(x, y)|<\epsilon,
$$

for $x \geq T$, we will say that solutions of Eq. (2.2) are uniformly globally attractive.

\section{Main results}

In this section we give two results for Eq. (1.1). The first one relies on the Darbo's fixed point theorem and the second one on the Mönch's fixed point theorem. Eq. (1.1) will be considered under the following assumptions :

- $\left(H_{1}\right)$ The function $f$ is continuous and there exists $b \in L^{1}\left(J, \mathbb{R}_{+}\right)$such that

$$
\left|f\left(x, y, u_{1}\right)-f\left(x, y, u_{2}\right)\right| \leq b(x, y)\left|u_{1}-u_{2}\right| ;(x, y) \in J ; u_{1}, u_{2} \in \mathbb{R} .
$$

- $\left(H_{2}\right)$ there exist continuous and bounded functions $h, g: J \rightarrow \mathbb{R}_{+}$such that

$$
\int_{1}^{x} \int_{1}^{y}\left(\ln \frac{x}{s}\right)^{r_{1}-1}\left(\ln \frac{y}{t}\right)^{r_{2}-1} b(t, s) d t d s \leq h(x, y)
$$

with

and

$$
k:=\frac{\|h\|}{\Gamma\left(r_{1}\right) \Gamma\left(r_{2}\right)}<1
$$

$$
\int_{1}^{x} \int_{1}^{y}\left(\ln \frac{x}{s}\right)^{r_{1}-1}\left(\ln \frac{y}{t}\right)^{r_{2}-1}|f(t, s, 0)| d t d s \leq g(x, y) .
$$

Remark 3.1. In view of the assumption $\left(H_{1}\right)$ we infer that for each $u \in \mathbb{R}$

$$
|f(x, y, u)| \leq b(x, y)|u|+|f(x, y, 0)| .
$$

Theorem 3.1. Under assumptions $\left(H_{1}\right)-\left(H_{2}\right)$ Eq. (1.1) has at least one solution $u=u(x, y)$ in the space $X$. Moreover, solutions of $E q$. (1.1) are globally attractive.

Proof. Consider the operator $F$ defined on the space $X$ by:

$$
(F u)(x, y)=\mu(x, y)+\frac{1}{\Gamma\left(r_{1}\right) \Gamma\left(r_{2}\right)} \int_{1}^{x} \int_{1}^{y}\left(\ln \frac{x}{s}\right)^{r_{1}-1}\left(\ln \frac{y}{t}\right)^{r_{2}-1} \frac{f(t, s, u(t, s))}{s t} d t d s .
$$

Observe that in view of our assumptions, for any function $u \in X$ the function $F u$ is continuous on $J$. Next, let us take an arbitrary function $u \in X$. Using our assumptions, for a fixed $(x, y) \in J$ we have

$$
\begin{aligned}
& |F u(x, y)| \\
& \leq|\mu(x, y)|+\frac{1}{\Gamma\left(r_{1}\right) \Gamma\left(r_{2}\right)} \int_{1}^{x} \int_{1}^{y}\left(\ln \frac{x}{s}\right)^{r_{1}-1}\left(\ln \frac{y}{t}\right)^{r_{2}-1} \frac{|f(t, s, u(t, s))|}{s t} d t d s \\
& \leq|\mu(x, y)|+\frac{1}{\Gamma\left(r_{1}\right) \Gamma\left(r_{2}\right)} \int_{1}^{x} \int_{1}^{y}\left(\ln \frac{x}{s}\right)^{r_{1}-1}\left(\ln \frac{y}{t}\right)^{r_{2}-1}[b(t, s)\|u\|+|f(t, s, 0)|] d t d s \\
& \leq|\mu(x, y)|+\frac{1}{\Gamma\left(r_{1}\right) \Gamma\left(r_{2}\right)} \int_{1}^{x} \int_{1}^{y}\left(\ln \frac{x}{s}\right)^{r_{1}-1}\left(\ln \frac{y}{t}\right)^{r_{2}-1} b(t, s)\|u\| d t d s \\
& +\int_{1}^{x} \int_{1}^{y}\left(\ln \frac{x}{s}\right)^{r_{1}-1}\left(\ln \frac{y}{t}\right)^{r_{2}-1}|f(t, s, 0)| d t d s .
\end{aligned}
$$


Hence we obtain

$$
\|F u\| \leq\|\mu\|+\frac{\|h\|\|u\|}{\Gamma\left(r_{1}\right) \Gamma\left(r_{2}\right)}+\|g\| .
$$

Thus, we infer that the function $F u$ is bounded on $J$. Then $F u \in X$.

Take

$$
r=\frac{\|\mu\|+\|g\|}{1-\frac{\|h\|}{\Gamma\left(r_{1}\right) \Gamma\left(r_{2}\right)}} .
$$

We deduce that the operator $F$ transforms the ball $B_{r}$ into itself.

Further, let $\left(u_{n}\right) \subset B_{r}$ such that $u_{n} \rightarrow u$ we get

$$
\begin{aligned}
& \left|F u_{n}(x, y)-F u(x, y)\right| \\
& \leq \frac{1}{\Gamma\left(r_{1}\right) \Gamma\left(r_{2}\right)} \int_{1}^{x} \int_{1}^{y}\left(\ln \frac{x}{s}\right)^{r_{1}-1}\left(\ln \frac{y}{t}\right)^{r_{2}-1} \frac{\left|f\left(t, s, u_{n}(x, y)\right)-f(t, s, u(x, y))\right|}{s t} d t d s \\
& \leq \frac{\left\|u_{n}-u\right\|}{\Gamma\left(r_{1}\right) \Gamma\left(r_{2}\right)} \int_{1}^{x} \int_{1}^{y}\left(\ln \frac{x}{s}\right)^{r_{1}-1}\left(\ln \frac{y}{t}\right)^{r_{2}-1} b(t, s) d t d s .
\end{aligned}
$$

Then

$$
\left\|F u_{n}-F u\right\| \leq \frac{\|h\|\left\|u_{n}-u\right\|}{\Gamma\left(r_{1}\right) \Gamma\left(r_{2}\right)} .
$$

Then when $n \rightarrow \infty$ we obtain $F u_{n} \rightarrow F u$ so $F$ is continuous on $B_{r}$.

Now, we take a nonempty $Y \subset B_{r}$, for $T \geq 1,\left(x_{1}, y_{1}\right),\left(x_{2}, y_{2}\right) \in[1, T] \times[1, b]$ with $\left|x_{2}-x_{1}\right| \leq \epsilon_{1}$ and $\left|y_{2}-y_{1}\right| \leq \epsilon_{2}$; for each $\epsilon_{1}, \epsilon_{2}>0$. Fix arbitrarily $u$ in $Y$ we have

$$
\begin{aligned}
& \left|F u\left(x_{2}, y_{2}\right)-F u\left(x_{1}, y_{1}\right)\right| \\
& =\mid \frac{1}{\Gamma\left(r_{1}\right) \Gamma\left(r_{2}\right)} \int_{1}^{x_{2}} \int_{1}^{y_{2}}\left(\ln \frac{x_{2}}{s}\right)^{r_{1}-1}\left(\ln \frac{y_{2}}{t}\right)^{r_{2}-1} \frac{f(t, s, u(s, t))}{s t} d t d s \\
& -\frac{1}{\Gamma\left(r_{1}\right) \Gamma\left(r_{2}\right)} \int_{1}^{x_{1}} \int_{1}^{y_{1}}\left(\ln \frac{x_{1}}{s}\right)^{r_{1}-1}\left(\ln \frac{y_{1}}{t}\right)^{r_{2}-1} \frac{f(t, s, u(s, t))}{s t} d t d s \mid \\
& \leq \frac{1}{\Gamma\left(r_{1}\right) \Gamma\left(r_{2}\right)}\left(x_{\left.x_{1}, y_{1}\right),\left(x_{2}, y_{2}\right) \in[1, T] \times[1, b]}\left|u\left(x_{2}, y_{2}\right)-u\left(x_{1}, y_{1}\right)\right|\right. \\
& \int_{1}^{x_{2}} \int_{1}^{y_{2}}\left(\ln \frac{x_{2}}{s}\right)^{r_{1}-1}\left(\ln \frac{y_{2}}{t}\right)^{r_{2}-1} b(t, s) d t d s \\
& \leq \frac{\|h\|}{\Gamma\left(r_{1}\right) \Gamma\left(r_{2}\right)} \\
& \sup _{\left(x_{1}, y_{1}\right),\left(x_{2}, y_{2}\right) \in[1, T] \times[1, b]}\left|u\left(x_{2}, y_{2}\right)-u\left(x_{1}, y_{1}\right)\right| .
\end{aligned}
$$

Thus

$$
\omega_{0}(F Y) \leq k \omega_{0}(Y) .
$$

Further, for $u, v \in Y$ and an arbitrary fixed $(x, y) \in[1, T] \times[1, b]$ we obtain

$$
\begin{aligned}
& |F u(x, y)-F v(x, y)| \\
& =\mid \frac{1}{\Gamma\left(r_{1}\right) \Gamma\left(r_{2}\right)} \int_{1}^{x} \int_{1}^{y}\left(\ln \frac{x}{s}\right)^{r_{1}-1}\left(\ln \frac{y}{t}\right)^{r_{2}-1} \frac{f(t, s, u(s, t))}{s t} d t d s \\
& -\frac{1}{\Gamma\left(r_{1}\right) \Gamma\left(r_{2}\right)} \int_{1}^{x} \int_{1}^{y}\left(\ln \frac{x}{s}\right)^{r_{1}-1}\left(\ln \frac{y}{t}\right)^{r_{2}-1} \frac{f(t, s, v(s, t))}{s t} d t d s \mid \\
& \leq \frac{1}{\Gamma\left(r_{1}\right) \Gamma\left(r_{2}\right)} \int_{1}^{x} \int_{1}^{y}\left(\ln \frac{x}{s}\right)^{r_{1}-1}\left(\ln \frac{y}{t}\right)^{r_{2}-1}|f(t, s, u(s, t))-f(t, s, v(s, t))| d t d s \\
& \leq \frac{1}{\Gamma\left(r_{1}\right) \Gamma\left(r_{2}\right)} \int_{1}^{x} \int_{1}^{y}\left(\ln \frac{x}{s}\right)^{r_{1}-1}\left(\ln \frac{y}{t}\right)^{r_{2}-1} b(t, s)|u(s, t)-v(s, t)| d t d s \\
& \leq \frac{\|h\|}{\Gamma\left(r_{1}\right) \Gamma\left(r_{2}\right)} \sup _{u, v \in Y}|u(s, t)-v(s, t)| .
\end{aligned}
$$


Then

$$
\lim _{x \longrightarrow \infty} \sup \operatorname{diam}(F Y)(x, y) \leq k \lim _{x \longrightarrow \infty} \sup \operatorname{diam} Y(x, y) .
$$

Observe, that linking (3.1), (3.2) and the definition of the measure of noncompactness $\psi$ given by the formula (2.1), we obtain

$$
\psi(F Y) \leq k \psi(Y) .
$$

Finally, in view of the Darbo fixed point theorem we deduce that $F$ has at least one fixed point in $B_{r}$ which is a solution of Eq. (1.1). Moreover, taking into account the fact that the set Fix $F \in k e r \psi$ and the characterization of sets belonging to ker $\psi$ (Remark 2.2) we conclude that all solutions of Eq. (1.1) are globally attractive in the sense of Definition 2.2.

Now we will formulate an other result by applying Mönch's Theorem.

Theorem 3.2. Under assumptions $\left(H_{1}\right)-\left(H_{2}\right)$ Eq. (1.1) has at least one solution $u=u(x, y)$ in the space $X$. Moreover, solutions of $E q$. (1.1) are globally attractive.

Proof. We have $F: B_{r} \rightarrow B_{r}$ continuous, let $Y \subset B_{r}$ with $Y=F(Y) \cup\{0\}$. Then for all $u$ in $Y$, there exist $v$ in $Y$ such that $u=F v$.

For $T \geq 1,\left(x_{1}, y_{1}\right),\left(x_{2}, y_{2}\right) \in[1, T] \times[1, b]$ such that $\left|x_{2}-x_{1}\right| \leq \epsilon_{1},\left|y_{2}-y_{1}\right| \leq \epsilon_{2}, \epsilon_{1}, \epsilon_{2}>0$ and $u, v \in Y$ we get

$$
\begin{aligned}
& \left|u\left(x_{2}, y_{2}\right)-u\left(x_{1}, y_{1}\right)\right| \\
& =\mid \frac{1}{\Gamma\left(r_{1}\right) \Gamma\left(r_{2}\right)} \int_{1}^{x_{2}} \int_{1}^{y_{2}}\left(\ln \frac{x}{s}\right)^{r_{1}-1}\left(\ln \frac{y}{t}\right)^{r_{2}-1} \frac{f(t, s, v(s, t))}{s t} d t d s \\
& -\frac{1}{\Gamma\left(r_{1}\right) \Gamma\left(r_{2}\right)} \int_{1}^{x_{1}} \int_{1}^{y_{1}}\left(\ln \frac{x}{s}\right)^{r_{1}-1}\left(\ln \frac{y}{t}\right)^{r_{2}-1} \frac{f(t, s, v(s, t))}{s t} d t d s \mid \\
& \leq \frac{\sup _{v \in A}\left|v\left(x_{2}, y_{2}\right)-v\left(x_{1}, y_{1}\right)\right|}{\Gamma\left(r_{1}\right) \Gamma\left(r_{2}\right)} \int_{1}^{x_{2}} \int_{1}^{y_{2}}\left(\ln \frac{x}{s}\right)^{r_{1}-1}\left(\ln \frac{y}{t}\right)^{r_{2}-1} b(t, s) d t d s .
\end{aligned}
$$

In view of our assumptions, we have

$$
\begin{aligned}
\sup _{u \in A}\left|u\left(x_{2}, y_{2}\right)-u\left(x_{1}, y_{1}\right)\right| & \leq \frac{\|h\|}{\Gamma\left(r_{1}\right) \Gamma\left(r_{2}\right)} \sup _{v \in A}\left|v\left(x_{2}, y_{2}\right)-v\left(x_{1}, y_{1}\right)\right|, \\
\lim _{\epsilon_{1}, \epsilon_{2} \rightarrow 0} \sup _{u \in A}\left|u\left(x_{2}, y_{2}\right)-u\left(x_{1}, y_{1}\right)\right| & \leq \frac{\|h\|}{\Gamma\left(r_{1}\right) \Gamma\left(r_{2}\right)} \lim _{\epsilon_{1}, \epsilon_{2} \rightarrow 0} \sup _{v \in A}\left|v\left(x_{2}, y_{2}\right)-v\left(x_{1}, y_{1}\right)\right| \\
\lim _{T \rightarrow \infty} \lim _{\epsilon_{1}, \epsilon_{2} \rightarrow 0} \sup _{u \in A}\left|u\left(x_{2}, y_{2}\right)-u\left(x_{1}, y_{1}\right)\right| & \leq \frac{\|h\|}{\Gamma\left(r_{1}\right) \Gamma\left(r_{2}\right)} \lim _{T \rightarrow \infty} \lim _{\epsilon_{1}, \epsilon_{2} \rightarrow 0} \sup _{v \in A}\left|v\left(x_{2}, y_{2}\right)-v\left(x_{1}, y_{1}\right)\right| .
\end{aligned}
$$

Then

$$
\omega_{0}(Y) \leq k \omega_{0}(Y)
$$

Next, let $u, v, w, z \in Y$ such that $u=F v$ and $w=F z$, for $x, y \in J$ we have

$$
\begin{aligned}
& |u(x, y)-w(x, y)| \\
& \leq \frac{1}{\Gamma\left(r_{1}\right) \Gamma\left(r_{2}\right)} \int_{1}^{x} \int_{1}^{y}\left(\ln \frac{x}{s}\right)^{r_{1}-1}\left(\ln \frac{y}{t}\right)^{r_{2}-1}|f(t, s, v(s, t))-f(t, s, z(s, t))| d t d s \\
& \leq \frac{\|h\|}{\Gamma\left(r_{1}\right) \Gamma\left(r_{2}\right)}|v(x, y)-z(x, y)| .
\end{aligned}
$$

Thus

Then

$$
\operatorname{diam} Y(x, y) \leq \frac{\|h\|}{\Gamma\left(r_{1}\right) \Gamma\left(r_{2}\right)} \operatorname{diam} Y(x, y)
$$

$$
\lim _{x \longrightarrow \infty} \sup \operatorname{diam}(Y)(x, y) \leq k \lim _{x \longrightarrow \infty} \sup \operatorname{diam} Y(x, y) .
$$

From the estimates (3.3) and (3.4) we infer that

$$
\psi(Y) \leq k \psi(Y) .
$$

Hence $k<1$, we obtain $\psi(Y)=0$. Combining the above result and Theorem 2.2 we complete the proof. 


\section{Example}

We consider the following Hadamard-Volterra integral equation

$$
u(x, y)=\frac{x+1}{y^{2} e^{x}}+\frac{1}{\Gamma\left(r_{1}\right) \Gamma\left(r_{2}\right)} \int_{1}^{x} \int_{1}^{y}\left(\ln \frac{x}{s}\right)^{r_{1}-1}\left(\ln \frac{y}{t}\right)^{r_{2}-1} \frac{e^{-t}+s}{t^{2} s^{3}+1} \sqrt{u^{2}(t, s)+e^{-t}} \frac{d t d s}{s t}
$$

where $(x, y) \in J=[1,+\infty) \times[1, b], b>1$ and $r_{1}, r_{2}>0$.

Set

$$
\mu(x, y)=\frac{x+1}{y^{2} e^{x}}, f(t, s, u(t, s))=\frac{e^{-t}+s}{t^{2} s^{3}+1} \sqrt{u^{2}(t, s)+e^{-t}} ;(t, s) \in J .
$$

It is clear that equation (4.1) can be written as equation (1.1). Let us show that conditions $\left(H_{1}\right)-\left(H_{3}\right)$ hold.

$$
\begin{aligned}
\left|f\left(t, s, u_{1}(t, s)\right)-f\left(t, s, u_{2}(t, s)\right)\right| & \leq \frac{e^{-t}+s}{t^{2} s^{3}+1}\left|\sqrt{u_{2}^{2}(t, s)+e^{-t}}-\sqrt{u_{1}^{2}(t, s)+e^{-t}}\right| \\
& \leq \frac{e^{-t}+s}{t^{2} s^{3}+1}\left|\sqrt{u_{2}^{2}(t, s)}-\sqrt{u_{1}^{2}(t, s)}\right| \\
& \leq \frac{e^{-t}+s}{t^{2} s^{3}+1}\left|u_{2}(t, s)-u_{1}(t, s)\right| .
\end{aligned}
$$

Take

We have

$$
b(t, s)=\frac{e^{-t}+s}{t^{2} s^{3}+1}, f(t, s, 0)=\frac{e^{-t}+s}{t^{2} s^{3}+1} e^{-\frac{1}{2} t} .
$$

$$
\begin{aligned}
& \int_{1}^{x} \int_{1}^{y}\left(\ln \frac{x}{s}\right)^{r_{1}-1}\left(\ln \frac{y}{t}\right)^{r_{2}-1} \frac{e^{-t}+s}{t^{2} s^{3}+1} d t d s \\
& \leq(\ln x)^{r_{1}}(\ln y)^{r_{2}} \int_{1}^{x} \int_{1}^{y} \frac{e^{-t}+s}{t^{2} s^{3}} d t d s \\
& =(\ln x)^{r_{1}}(\ln y)^{r_{2}} \int_{1}^{x} \int_{1}^{y}\left(\frac{e^{-t}}{s^{3}}+\frac{1}{t^{2} s^{2}}\right) d t d s \\
& =(\ln x)^{r_{1}}(\ln y)^{r_{2}}\left[\left(-e^{-y}+e^{-1}\right)\left(-\frac{1}{2 x^{2}}+\frac{1}{2}\right)+\left(-\frac{1}{y}+1\right)\left(-\frac{1}{x}+1\right)\right] \\
& =h(x, y),
\end{aligned}
$$

and

$$
\begin{aligned}
& \int_{1}^{x} \int_{1}^{y}\left(\ln \frac{x}{s}\right)^{r_{1}-1}\left(\ln \frac{y}{t}\right)^{r_{2}-1}|f(t, s, 0)| d t d s \\
& \leq(\ln x)^{r_{1}}(\ln y)^{r_{2}} \int_{1}^{x} \int_{1}^{y} \frac{e^{-t}+s}{t^{2} s^{3}+1} e^{-\frac{1}{2} t} d t d s \\
& \leq(\ln x)^{r_{1}}(\ln y)^{r_{2}} \int_{1}^{x} \int_{1}^{y} \frac{e^{-t}+s}{t^{2} s^{3}} e^{-\frac{1}{2} t} d t d s \\
& =(\ln x)^{r_{1}}(\ln y)^{r_{2}} \int_{1}^{x} \int_{1}^{y}\left(\frac{e^{-\frac{3}{2} t}}{s^{3}}+\frac{e^{-\frac{1}{2} t}}{s^{2}}\right) d t d s \\
& =(\ln x)^{r_{1}}(\ln y)^{r_{2}}\left[\left(-\frac{2}{3} e^{-\frac{3}{2} y}+\frac{2}{3} e^{-\frac{3}{2}}\right)\left(-\frac{1}{2 x^{2}}+\frac{1}{2}\right)\right. \\
& \left.+\left(-\frac{1}{x}+1\right)\left(-2 e^{-\frac{1}{2} y}+2 e^{-\frac{1}{2}}\right)\right]=g(x, y) .
\end{aligned}
$$

It is clear that $h(x, y), g(x, y)$ are bounded functions on $J$. And we have also

$$
k=\frac{\|h\|}{\Gamma\left(r_{1}\right) \Gamma\left(r_{2}\right)}=\frac{\frac{1}{2 e}+1}{\Gamma\left(r_{1}\right) \Gamma\left(r_{2}\right)}<1,
$$

for an arbitrary $r_{1}, r_{2}$. Then from Theorem 3.1 the Eq. (4.1) has at least solution in $X$ and solutions of Eq. (4.1) are globally attractive. 


\section{References}

[1] Abbas, S., Benchohra, M., Graef, J. and Henderson, J., Implicit Fractional Differential and Integral Equations; Existence and Stability, De Gruyter, Berlin, 2018.

[2] Abbas, S., Benchohra, M., and N'Guérékata, G. M., Topics in Fractional Differential Equations, Springer, New York, 2012.

[3] Abbas, S., Benchohra, M. and N'Guérékata, G. M., Advanced Fractional Differential and Integral Equations, Nova Science Publishers, New York, 2015.

[4] Abbas, S., Benchohra, M. and Henderson, J., Global asymptotic stability of solutions of nonlinear quadratic Volterra integral equations of fractional order Comm. Appl. Nonlinear Anal.19 (2012), no. 1, 79-89.

[5] Abbas, S., Alaidarous, E., Benchohra, M. and Nieto, J.J., Existence and stability of solutions for HadamardStieltjes fractional integral equations. Discrete Dyn. Nat. Soc. 2015, Art. ID 317094, 6 pp.

[6] Appell, J., Measure of noncompactness, condensing operators and fixed points: an application-oriented survey Fixed Point Theory, 6 (2) (2005), 157-229.

[7] Agarwal, R.P. and O'Regan, D., Infinite Interval Problems for Differential, Difference and Integral Equations Kluwer Academic Publishers, Dordrecht, 2001.

[8] Ahmad, B. and Ntouyas, S.K., Initial value problems of fractional order Hadamard-Type functional differential equations Electron. J. Differential Equations 2015, No. 77, 9 pp.

[9] Banas, J. and O'Regan, D., On existence and local attractivity of solutions of a quadratic Volterra integral equations of fractional order J. Math. Anal. Appl. 345 (2008), no. 1, 573-582.

[10] Banas, J. and Rzepka, B., Nondecreasing solutions of a quadratic singular Volterra integral equation Math. Comput. Modelling 49 (2009), no. 3-4, 488-496.

[11] Banas, J., Rocha Martin, J. and Sadarangani, K., On solutions of a quadratic integral equations of Hammerstein type Math. Comput. Modelling 43 (2006), no. 1-2, 97-104.

[12] Banas, J., Rocha Martin, J. and Sadarangani, K., On existence and asymptotic stability of solutions of a nonlinear integral equation J. Math. Anal. Appl. 284 (2003), no. 1, 165-173.

[13] Banas, J., Caballero, J., Rocha, J. and Sadarangani, K., Global asymptotic stability of solutions of a functional integral equation Nonlinear Anal. 69 (2008), no. 7, 1945-1952.

[14] Banas, J. and Dhage, B.C., Monotonic solutions of a class of quadratic integral equations of Volterra type Comput. Math. Appl. 49 (2005), no. 5-6, 943-952.

[15] Banas, J. and Rzepka, B., On existence and asymptotic stability of solutions of a nonlinear integral equation $J$. Math. Anal. Appl 284 (2003), no. 1, 165-173.

[16] Banas, J., Cabrera, J. and Sadarangani, K., On existence and asymptotic behaviour of solutions of a functional integral equation Nonlinear Anal. 66 (2007), no. 10, 2246-2254.

[17] Baskonus, H. M. and Bulut, H., On the numerical solutions of some fractional ordinary differential equations by fractional Adams-Bashforth-Moulton method. Open Math. 13 (2015), 547-556.

[18] Benchohra, M., Henderson, J. and Seba, D., Measure of noncompactness and fractional differential equations in Banach spaces, PanAmer. Math. J. 20 (2010), no. 3, 27-37.

[19] Corduneanu, C., Integral Equations and Applications Cambridge Univ. Press, Cambridge, 1991.

[20] González, C., Melado, A.J. and Fuster, E.L., A Mönch type fixed point theorem under the interior condition J. Math. Anal. Appl. 352 (2009), 816-821.

[21] Katugampola, U., New approach to generalized fractional integral Appl. Math. Comput. 218 (2011), no. 3, 860-865. 
[22] Kilbas, A., Hadamard-Type fractional calculus, J. Korean Math. Soc. 38 (2001), no. 6, 1191-1204.

[23] Samko, S., Kilbas, A. and Marichev, O.I., Fractional Integrals and Derivatives (Theorie and Applications) Gordon and Breach Science Publishers. Yverdon, 1993.

[24] Thiramanus, P., Tariboon, J. and Ntouyas, S.K., Integrals inequalities with 'maxima' and their applications to Hadamard-Type fractional differential equations J. Inequal. Appl. 2014, 2014:398, 15 pp.

\section{Affiliations}

SAID BAGHDAD

ADDRESS: Laboratory of Mathematics, Djillali Liabes University of Sidi Bel-Abbès, P.O. Box 89, Sidi Bel-Abbès 22000, Algeria.

E-MAIL: said_b@gymail.com

ORCID ID: 0000-0002-2861-5256

MOUFFAK BENCHOHRA

AdDRESS: Laboratory of Mathematics, Djillali Liabes University of Sidi Bel-Abbès, P.O. Box 89, Sidi Bel-Abbès 22000, Algeria, and Department of Mathematics, College of Science, King Saud University, P.O. Box 2455, Riyadh 11451, Saudi Arabia.

E-MAIL: benchohra@yahoo.com

ORCID ID: 0000-0003-3063-9449 